This is a postprint version of the following published document:

Fernández-Galisteo, Daniel; Jiménez, Carmen; Sánchez-Sanz, Mario; Kurdyumov, Vadim N. The differential diffusion effect of the intermediate species on the stability of premixed flames propagating in microchannels. Combustion Theory and Modelling 18 (2014) 4-5, pp. 582-605. Avalaible in http://dx.doi.org/10.1080/13647830.2014.946970

(C) 2014 Taylor \& Francis 


\title{
The differential diffusion effect of the intermediate species on the stability of premixed flames propagating in microchannels
}

\author{
Daniel Fernández-Galisteo ${ }^{\mathrm{a} *}$, Carmen Jiménez $^{\mathrm{a}}$, Mario Sánchez-Sanz ${ }^{\mathrm{b}}$ and \\ Vadim N. Kurdyumov ${ }^{a}$ \\ ${ }^{a}$ Department of Energy, Centro de Investigaciones Energéticas, Medioambientales y Tecnológicas \\ (CIEMAT), Avda. Complutense 40, 28040 Madrid, Spain; ${ }^{b}$ Departamento Ingeniería Térmica y de \\ Fluidos, Universidad Carlos III de Madrid, 28911, Leganés, Spain
}

\begin{abstract}
The propagation of premixed flames in adiabatic and non-catalytic planar microchannels subject to an assisted or opposed Poiseuille flow is considered. The diffusive-thermal model and the well-known two-step chain-branching kinetics are used in order to investigate the role of the differential diffusion of the intermediate species on the spatial and temporal flame stability. This numerical study successfully compares steady-state and time-dependent computations to the linear stability analysis of the problem. Results show that for fuel Lewis numbers less than unity, $L e_{F}<1$, and at sufficiently large values of the opposed Poiseuille flow rate, symmetry-breaking bifurcation arises. It is seen that small values of the radical Lewis number, $L e_{Z}$, stabilise the flame to symmetric shape solutions, but result in earlier flashback. For very lean flames, the effect of the radical on the flame stabilisation becomes less important due to the small radical concentration typically found in the reaction zone. Cellular flame structures were also identified in this regime. For $L e_{F}>1$, flames propagating in adiabatic channels suffer from oscillatory instabilities. The Poiseuille flow stabilises the flame and the effect of $L e_{Z}$ is opposite to that found for $L e_{F}<1$. Small values of $L e_{Z}$ further destabilise the flame to oscillating or pulsating instabilities.
\end{abstract}

Keywords: premixed flames dynamics; chain-branching kinetic model; microcombustion; symmetry-breaking bifurcation; oscillatory instability

\section{Introduction}

The aim of the present work is to study the influence of the intermediate species on the stabilisation and dynamics of flame propagation in adiabatic and non-catalytic microchannels. The systematic study of the intermediate species effect can provide insight into the design and operation of practical microcombustors by reducing the undesirable unsteady combustion modes. The role of the intermediate species could be of particular interest in the catalytic combustion [1] used in micro-scale reactors coupled, for example, with fuel reforming for hydrogen production $[2,3]$.

Most theoretical studies of flame dynamics in channels have employed a simple one-step Arrhenius-type model $F+O \rightarrow P$, where $F, O$ and $P$ denote the fuel, the oxidant and the products, respectively, for chemistry modelling. Although this simple kinetic model does not consider intermediate species, it has demonstrated an acceptable success in clarifying the role of the complex physical phenomena involved in flame dynamics in channels [4-11].

*Corresponding author. Email: d.galisteo@ciemat.es 
Among the most important phenomena, we distinguish the flame-fluid or momentum interaction (where we can include the thermal expansion and viscous effect), the flamewall interaction (including both the thermal and catalytic interaction), and the differential diffusion effect.

A one-step model was used in [4] for predicting the flashback conditions and flame shapes in an adiabatic planar channel for $L e_{F}=1$ and subject to a prescribed Poiseuille flow. $L e_{F} \neq 1$ flames were further studied in [6], also in adiabatic channels and with the use of a one-step kinetic model. There, it was demonstrated that for $L e_{F}<1$ the differential diffusion effect destabilises the steady symmetric solutions to non-symmetric ones, this effect being amplified due to flame curvature when the flow rate in the channel is increased. Only non-symmetric solutions remain stable for large enough values of the flow rate, following a supercritical bifurcation. These non-symmetric solutions modify the flashback condition. On the other hand, for $L e_{F}>1$, in cases where the planar flame suffers from oscillatory instabilities [12], the Poiseuille flow stabilises the flame and oscillatory solutions vanish. The effect of the differential diffusion between heat and mass in the symmetry-breaking or oscillatory instability occurrence is seen to decrease in importance as the width of the channel is reduced [6,7]. All the work cited above made use of the thermo-diffusive approximation, also called the constant density approximation, to eliminate the flame-fluid interaction due to thermal expansion and viscous effects and therefore to concentrate on the preferential diffusion effect of the fuel.

When the thermal flame-wall interaction effect is included, the flame dynamics become richer. Heat loss is found to amplify the diffusive-thermal instability for $L e_{F}<1$, as demonstrated in a Hele-Shaw configuration $[13,14]$, for example, can also drive the flame to oscillate $[15,16]$. These unsteady solutions were found when increasing the duct size in the case of isothermal walls for $L e_{F}<1$ [8]; however, they only appear in the adiabatic-wall cases for $L e_{F}>1$, as shown later in the present work. Following a subcritical Hopf bifurcation, two stable combustion modes, that is, a steady weak flame (also called mild combustion) and an ignition-extinction oscillating flame, can coexist when the wall temperature is sufficiently close to the quenching extinction limit. When the channel height increases, a more complex dynamic arises: two oscillatory modes, symmetric and non-symmetric, can coexist; see [9]. Different burning modes such as mild combustion, ignition-extinction, steady symmetric and non-symmetric flames, and oscillating or pulsating flames, including cellular and chaotic behaviour, have been identified in the numerical simulation of premixed hydrogen-air flames in micro and meso channels with a specified wall temperature and using detailed chemistry and transport [17,18]. A complex combustion mode, called a spinning flame, where the flame presents a non-symmetric shape and rotates in the azimuthal direction, has also been reported recently in three-dimensional studies of hydrogen-air flames in tubes [19]. In these simulations it was observed that the lighter species or radicals $(\mathrm{H}, \mathrm{O}, \mathrm{OH})$ were the first species to move away from the tube axis when increasing the inlet velocity in an axisymmetric flame solution, anticipating the role of radicals in flame stability, as discussed below in Section 6.

Some combustion modes obtained in numerical simulations were first observed experimentally. For instance, stationary and repetitive ignition-extinction flames were stabilised in both a fixed-wall-temperature configuration and without an imposed wall-temperature distribution, in methane and/or hydrogen-air mixtures for medium and low flow rates, respectively; see [20-23].

Based on the diffusive-thermal model and using a one-step kinetic model, Daou and Matalon [5] studied the effect of heat loss to the walls in extinction conditions for steady propagation, obtaining a value of the quenching distance in agreement with the experimental 
one. The effect of heat recirculation in extinction conditions was studied for a simplified two-dimensional steady Swiss-roll-like model $[10,11]$ and using quasi-one-dimensional analysis [24]. Heat recirculation can significantly extend the flammability limit and the quenching distance.

When the catalytic flame-wall interaction is taken into account, much of the oscillating and steady non-symmetric combustion modes predicted in planar channels can be suppressed, as obtained for example for hydrogen-air flames with platinum-coated walls [25]. The radical quenching and the heat generated by the surface recombination reactions at the walls, coupling with the gas mixture, were studied in [26] for a hydrogen-air impinging-jet configuration, demonstrating the importance of catalytic reactions in the ignition-extinction points, specially near flammability conditions. All of the above indicates that the reactivity of intermediate species plays a sufficiently important role in the flame dynamics in channels to justify a separate study, as done recently with the use of a two-step kinetic model in a quasi-one-dimensional study [27].

Therefore, before extending the one-step model results to a detailed chemistry, as done in [17-19] for premixed hydrogen-air flames, or to a global reaction, as in [28-30] for methane-air flames, the present work addresses the influence of the preferential diffusion of the intermediate species on the flame dynamics by using a simple two-step chainbranching model. This chain-branching kinetics is usually preferred as a better approach to real hydrocarbons and hydrogen flame description than that of the simple one-step model [31-33].

\section{Formulation of the problem}

Consider a premixed flame propagating in a planar channel. The fuel-air mixture is at initial temperature $T_{0}$ and immersed in a Poiseuille flow with mean velocity $U_{0}$. In what follows, $h$ denotes the height of the channel and $x^{\prime}, y^{\prime}$ denote the longitudinal and wall-normal coordinates, respectively. The resulting curved flame separates the fresh mixture, far to the left, from the combustion products downstream to the right, as shown in the sketch in Figure 1.

This paper deals with a diffusive-thermal model, so the density of the mixture $\rho$, the heat capacity $c_{p}$, the thermal diffusivity $\mathcal{D}_{T}$, and the molecular diffusivity of the fuel $\mathcal{D}_{F}$ and radical $\mathcal{D}_{Z}$ are all assumed to be constant. As a consequence, the flow field is not affected by combustion. For the small value of $h$ studied in the present work, which corresponds to the micro-scale combustion regime, the Reynolds number will be at most a few hundred [34] and the flow can then be considered primarily laminar. The flow velocity is given by the fully-developed Hagen-Poiseuille base flow, that is, $U\left(y^{\prime}\right)=u_{x^{\prime}}=6 U_{0}\left(1-y^{\prime} / h\right)\left(y^{\prime} / h\right)$

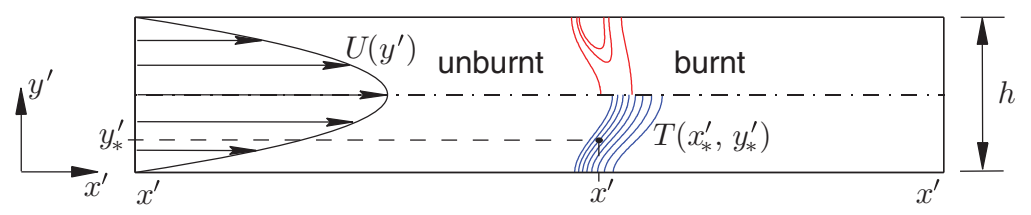

Figure 1. Sketch of the problem indicating the coordinate system, the Poiseuille base flow $U\left(y^{\prime}\right)$ and the typical symmetric tulip-shaped flame [28], marked here by the mass fraction contours of the intermediate species (upper half) and the isotherms (lower half). Also shown is an example (lower half) of the selected reference temperature $T_{*}=T\left(x_{*}^{\prime}, y_{*}^{\prime}\right)$, which is used to write Equations (3)-(5) in a moving reference frame. 
and $u_{y^{\prime}}=0$, where $u_{x^{\prime}}$ and $u_{y^{\prime}}$ denote the $x$ - and $y$-components of the flow velocity, respectively, so the transverse convective term does not enter into the formulation below.

The mixture is assumed to be deficient in fuel and therefore the mass fraction of the oxidant, which is in abundance, does not appear in the formulation. In order to evaluate the effect of the intermediate species, the chemical reaction is modelled by the chain-branching kinetics proposed by Dold et al. [31,32]. This two-step model includes the branching and recombination steps

$$
\begin{array}{cl}
F+Z \rightarrow 2 Z & : \quad \omega_{B}=A_{B} \frac{\rho Y_{F}}{W_{F}} \frac{\rho Y_{Z}}{W_{Z}} \exp \left\{-E / R^{o} T\right\}, \\
Z+M \rightarrow P+M+Q \quad: \quad \omega_{C}=A_{C} \frac{\rho Y_{Z}}{W_{Z}} \frac{\rho}{W}
\end{array}
$$

where $\omega_{B}$ is the molar rate per unit volume of the chain-branching reaction, which is thermally sensitive to the activation energy $E$, and $\omega_{C}$ is the molar rate per unit volume of the chain-termination reaction, which is independent of the temperature. In what follows, $A_{B}$ and $A_{C}$ are the frequency factors, $T$ is the temperature, $Z$ denotes the intermediate species, $Y_{F}$ and $Y_{Z}$ are the mass fractions of fuel and radical, $M$ stands for any third body, $R^{o}$ is the universal gas constant, and $W_{F}, W_{Z}$ and $W$ are the fuel, radical and mean molecular weights, respectively. All the heat $Q$ is assumed to be released only in the completion step, where $Q$ is the heat of combustion per unit mole of fuel.

The earliest chain-branching kinetic model, proposed by Zel'dovich and explored later by Liñán in the frame of hydrogen-oxygen combustion [35,36], assumed that the chaintermination reaction was second order in $Z$, that is, $2 Z+M \rightarrow P+M$. This former model suffered from the 'cold-boundary difficulty' [37] in the sense that branching is possible for all low concentrations of $Z$. Therefore, it is not possible to identify a temperature below which branching of intermediate species is cut off, as seen to occur in hydrogen chemistry [38].

Dold's model is more convenient. It provides the existence of this cut-off in the branching of radicals at a well-known crossover temperature. At this temperature branching and recombination of intermediate species balance in a homogeneous system, that is, $\omega_{B}=$ $\omega_{C}$. However, in a non-homogeneous system like a flame, the definition of the branching temperature $T_{c}$ turns out to be more useful. This branching temperature, also known as a non-homogeneous crossover temperature, is located in the thin reactive zone, where the concentration of $Z$ peaks, and is usually defined by the relation $\omega_{B}=\beta^{2} \omega_{C}$. In this inner zone, both recombination and diffusion enter to remove the radicals and compete with branching. By using an order-of-magnitude analysis, it is possible to demonstrate that a factor $\beta^{2}$ is needed to account for these effects. Another characteristic of the chainbranching kinetics is that fuel leakage can arise for flames close to the flammability limit, as demonstrated in [39]. The reader is referred to Dold et al. [31,32] for further details. From the latter expression, the branching temperature can be evaluated at the initial fuel mass fraction $Y_{F_{0}}$ and leads to the following implicit equation:

$$
\frac{A_{B}}{A_{C}} \frac{W}{W_{F}} Y_{F_{0}}=\left(\frac{E}{R^{o}} \cdot \frac{T_{c}-T_{0}}{T_{c}^{2}}\right)^{2} \exp \left\{\frac{E}{R^{o} T_{c}}\right\},
$$

to be used in the dimensionless formulation given below. Herein $\beta=E\left(T_{c}-T_{0}\right) / R^{o} T_{c}^{2}$ stands for the relevant Zel'dovich number based on $T_{c}$. 
The chain-branching model defines a kinetically controlled flammability limit, as occurs in the kinetic mechanism of hydrogen-air deflagrations [40,41]. Close to the flammability limit, a parallel between the two-step chain-branching kinetics and the one-step model emerges, with an effective activation energy in the one-step model that becomes singular as the flammability limit is approached [32,33,40,41].

The chain-branching reactions are located in the high-temperature region of the flame because of their typical high activation energy. These reactions produce an increase of the intermediate species or chemical radicals which diffuse upstream and downstream of this thin branching zone and recombine to products by means of a very exothermic chaintermination reaction in a wider region. Heat release occurs throughout the flame due to radical recombination, and fuel exhaustion is reached interior to the flame, which is a characteristic of real flames [32]. In the one-step model, however, fuel consumption and the final adiabatic flame temperature are reached simultaneously.

It is interesting to note that the two forms of instability of freely propagating flames, that is, cellular and oscillatory instabilities, are also found when using the two-step chainbranching kinetics. These instabilities depend mainly on $L e_{F}$ and the radical Lewis number, $L e_{Z}$, and also on the heat of reaction. However, the dependence of the solutions with the activation energy of the branching reaction is known to be weak, a surprising characteristic that differs from the behaviour of the one-step model. For $L e_{F}>1$, the chain-branching model predicts oscillatory instability, but only below a threshold heat of reaction, found to be very close to the flammability limit and for too high $L e_{F}[32,33,42]$. The radical concentration at this limit is very small, indicating the significant role of the intermediate species on flame stabilisation. Cellular instability arises, however, in a wider range of heats of reaction far from the flammability limit when $L e_{F}<1$.

As done before in $[6,8]$, a reference frame attached to the flame front at a point $\left(x_{*}^{\prime}, y_{*}^{\prime}\right)$ is used to describe the flame propagation. Consider a line parallel to the wall located at a distance $y^{\prime}=y_{*}^{\prime}$, as sketched in Figure 1. Following the temperature distribution along this line, starting from the unburned side, we choose the first point $x^{\prime}=x_{*}^{\prime}$ where the temperature is equal to some reference value $T=T_{*}$. We choose the reference frame moving at the velocity $U_{f}\left(t^{\prime}\right)$ of this point relative to the wall. Note that for steady flame propagation the whole flame surface moves with a constant velocity equal to $U_{f}$ independent of the location of the reference point $\left(x_{*}^{\prime}, y_{*}^{\prime}\right)$. However, for unsteady flame dynamics, the flame does not move as a whole and each point propagates with its own velocity. The specific form of $U_{f}$ depends in this case on the choice of the reference temperature $T_{*}$.

The conservation of energy and mass fraction equations written in the moving reference frame become

$$
\begin{gathered}
\frac{\partial T}{\partial t^{\prime}}+\left\{U_{f}\left(t^{\prime}\right)+6 U_{0} \frac{y^{\prime}}{h}\left(1-\frac{y^{\prime}}{h}\right)\right\} \frac{\partial T}{\partial x^{\prime}}=\mathcal{D}_{T} \Delta^{\prime} T+\frac{Q}{c_{p}} \cdot \frac{\omega_{C}}{\rho} \\
\frac{\partial Y_{F}}{\partial t^{\prime}}+\left\{U_{f}\left(t^{\prime}\right)+6 U_{0} \frac{y^{\prime}}{h}\left(1-\frac{y^{\prime}}{h}\right)\right\} \frac{\partial Y_{F}}{\partial x^{\prime}}=\mathcal{D}_{F} \Delta^{\prime} Y_{F}-W_{F} \frac{\omega_{B}}{\rho} \\
\frac{\partial Y_{Z}}{\partial t^{\prime}}+\left\{U_{f}\left(t^{\prime}\right)+6 U_{0} \frac{y^{\prime}}{h}\left(1-\frac{y^{\prime}}{h}\right)\right\} \frac{\partial Y_{Z}}{\partial x^{\prime}}=\mathcal{D}_{Z} \Delta^{\prime} Y_{Z}+W_{Z} \frac{\omega_{B}}{\rho}-W_{F} \frac{\omega_{C}}{\rho},
\end{gathered}
$$

where $\Delta^{\prime}=\partial^{2} / \partial x^{\prime 2}+\partial^{2} / \partial y^{\prime 2}$ and $t^{\prime}$ denotes the dimensional time.

To better clarify the problem, the burning velocity of the planar flame $S_{L}$ together with the thermal flame thickness, defined as $\delta_{T}=\mathcal{D}_{T} / S_{L}$, are used below to specify 
the dimensionless parameters. A non-dimensional temperature defined as $\theta=\left(T-T_{0}\right)$ / $\left(T_{c}-T_{0}\right)$ is also introduced in the following. Choosing $h$ and $h^{2} / \mathcal{D}_{T}$ as the reference length and timescales, the non-dimensional coordinates and time become respectively $(x, y)=$ $\left(x^{\prime} / h, y^{\prime} / h\right)$ and $t=t^{\prime} /\left(h^{2} / \mathcal{D}_{T}\right)$. Fuel and radical mass fraction are scaled with the initial mass fraction $Y_{F_{0}}$ according to $F=Y_{F} / Y_{F_{0}}$ and $Z=Y_{Z} W_{F} /\left(Y_{F_{0}} W_{Z}\right)$. Introducing these variables and making use of (2) reduces (3)-(5) to the dimensionless form

$$
\begin{gathered}
\frac{\partial \theta}{\partial t}+\sqrt{d}\left\{u_{f}(t)+6 m y(1-y)\right\} \frac{\partial \theta}{\partial x}=\Delta \theta+d \mu q Z \\
\frac{\partial F}{\partial t}+\sqrt{d}\left\{u_{f}(t)+6 m y(1-y)\right\} \frac{\partial F}{\partial x}=\frac{1}{L e_{F}} \Delta F-d \mu k(\theta) F Z \\
\frac{\partial Z}{\partial t}+\sqrt{d}\left\{u_{f}(t)+6 m y(1-y)\right\} \frac{\partial Z}{\partial x}=\frac{1}{L e_{Z}} \Delta Z+d \mu k(\theta) F Z-d \mu Z,
\end{gathered}
$$

where

$$
k(\theta)=\beta^{2} \exp \left\{\frac{\beta(\theta-1)}{1+\gamma(\theta-1)}\right\}
$$

is the dimensionless form of the chain-branching reaction rate constant $A_{B} \exp \left\{-E / R^{o} T\right\}$ given in (1). The value of the dimensionless burning velocity $u_{f}(t)=U\left(t^{\prime}\right) / S_{L}$ is calculated by the additional condition $\theta\left(x_{*}, y_{*} ; t\right)=\theta_{*}$, where $\theta_{*}=\left(T_{*}-T_{0}\right) /\left(T_{c}-T_{0}\right)$ is the dimensionless reference temperature at the reference point $\left(x_{*}, y_{*}\right)$. Typical values used during our calculations were $y_{*}=0.3$ and $\theta_{*}=0.8$, but different values could be used depending on $L e_{F}$.

Equations (6)-(9) need to be supplemented by the following boundary conditions far upstream and downstream of the flame front:

$$
\begin{array}{ll}
x \rightarrow-\infty: & \theta=F-1=Z=0, \\
x \rightarrow+\infty: & \partial \theta / \partial x=\partial F / \partial x=\partial Z / \partial x=0,
\end{array}
$$

and by the adiabatic and non-catalytic reactivity boundary conditions at the wall

$$
y=0 \quad \text { and } \quad y=1: \quad \partial \theta / \partial y=\partial F / \partial y=\partial Z / \partial y=0 .
$$

Initial conditions for Equations (6)-(9) in time-dependent calculations were chosen in the form of a hot spot for the temperature and the intermediate species, and a uniform distribution for the fuel mass fraction, such as

$$
t=0:\left\{\begin{array}{l}
\theta=\theta_{s} \exp \left\{-\left[\left(x-x_{s}\right)^{2}+\left(y-y_{s}\right)^{2}\right] / r_{s}^{2}\right\} \\
Z=Z_{s} \exp \left\{-\left[\left(x-x_{s}\right)^{2}+\left(y-y_{s}\right)^{2}\right] / r_{s}^{2}\right\} \\
F=1
\end{array}\right.
$$

where $\left(x_{s}, y_{s}\right)$ corresponds to the hot spot location and $r_{s}$ to its radius. Typical values of the parameters were taken as $\theta_{s}=1.2, Z_{s}=1, r_{s}=0.3, x_{s}=0.5$ and $y_{s}=0.3$. Note that this chain-branching kinetic model (1) cannot be used for modelling ignition processes for lack of the corresponding chain-initiation step, for instance $F \rightarrow Z$. In order to modelate 
the ignition-like problem herein a finite quantity of radicals $Z$ is necessary in the initial condition (12).

The following parameters appear in the above formulation: the heat release parameter $\gamma=\left(T_{c}-T_{0}\right) / T_{c}$, the dimensionless heat of reaction $q=Q Y_{F_{0}} /\left[c_{p}\left(T_{c}-T_{0}\right) W_{F}\right]$, the dimensionless mass flow rate $m=U_{0} / S_{L}$, and the reduced Damköhler number as a quotient of diffusion to chemical times $d=h^{2} S_{L}^{2} / \mathcal{D}_{T}^{2}$. The Damköhler number can also be expressed as $d=\left(h / \delta_{T}\right)^{2}$, the square of the ratio of the channel height to the thermal flame thickness.

The factor $\mu=\left(\rho A_{c} \mathcal{D}_{T}\right) /\left(W S_{L}^{2}\right)$ represents the reduced inverse square of the planar flame velocity and it is introduced herein to ensure that $u_{f}$ for a planar adiabatic flame equals one. The value of $\mu$ corresponds to the solution of the following one-dimensional eigenvalue problem:

$$
\begin{aligned}
\frac{\mathrm{d} \theta}{\mathrm{d} \xi} & =\frac{\mathrm{d}^{2} \theta}{\mathrm{d} \xi^{2}}+\mu q Z, \\
\frac{\mathrm{d} F}{\mathrm{~d} \xi} & =L e_{F}^{-1} \frac{\mathrm{d}^{2} F}{\mathrm{~d} \xi^{2}}-\mu \beta^{2} \exp \left\{\frac{\beta(\theta-1)}{1+\gamma(\theta-1)}\right\} F Z, \\
\frac{\mathrm{d} Z}{\mathrm{~d} \xi} & =L e_{Z}^{-1} \frac{\mathrm{d}^{2} Z}{\mathrm{~d} \xi^{2}}+\mu \beta^{2} \exp \left\{\frac{\beta(\theta-1)}{1+\gamma(\theta-1)}\right\} F Z-\mu Z,
\end{aligned}
$$

where $\xi=x \sqrt{d}$, together with the boundary conditions far upstream and downstream

$$
\begin{array}{ll}
\xi \rightarrow-\infty: & \theta=F-1=Z=0 \\
\xi \rightarrow+\infty: & \partial \theta / \partial \xi=\partial F / \partial \xi=\partial Z / \partial \xi=0 .
\end{array}
$$

The solution of the planar problem (13) and (14), which is required before solving the complete problem (6)-(11), was calculated numerically in [39]. For completeness, the variation of this factor $\mu$ with $q$ is shown in Figure 2 for different values of $L e_{F}$ and $L e_{Z}=1$.

In the calculations, the Zel'dovich number was fixed at $\beta=5$ as representative of combustion processes, which also corresponds to the value reported earlier for hydrogen chemistry [43]. The heat release parameter was kept fixed at $\gamma=0.7$.

Anticipating the numerical results, only steady symmetric and steady non-symmetric flames appear for $L e_{F}<1$. The steady-state computations $-\partial / \partial t=0$ in Equations (6)-(8)identified the bifurcation points where the flame breaks its symmetry. In order to check these bifurcation points, a linear stability analysis of the steady-state symmetric solution was also implemented, as explained below in Section 4. To obtain the steady-state symmetric base solution we forced the symmetry of the flame, even in cases when the flame is naturally non-symmetric, by reducing the domain to half of its height, $0 \leq y \leq 1 / 2$, and imposing the boundary conditions $\partial \theta / \partial y=\partial F / \partial y=\partial Z / \partial y=0$ at $y=1 / 2$ for the temperature and species mass fraction, respectively.

As done before in $[6,9]$, we also introduce herein the parameter

$$
S=\int_{-\infty}^{\infty}\left[\int_{0}^{1 / 2}\{\theta(x, y ; t)-\theta(x, 1-y ; t)\} \mathrm{d} y\right] \mathrm{d} x
$$

to evaluate the symmetry of the flame. For symmetric flames, $S=0$ within numerical accuracy, but it takes non-zero values when the solution becomes non-symmetric.

A time-dependent code solving the complete problem (6)-(12) was also implemented. The time-dependent computations and the stability analysis demonstrated that only steady 


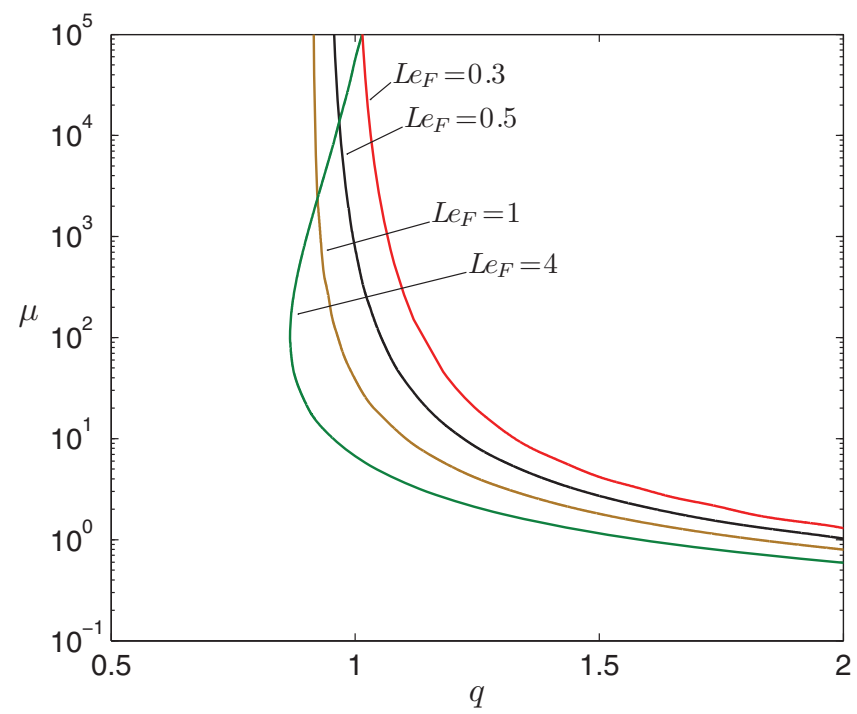

Figure 2. The variation of the eigenvalue $\mu$ with the dimensionless heat of reaction $q$ for the onedimensional problem defined in (13) and (14), for different $L e_{F}$ and calculated for $L e_{Z}=1, \beta=5$ and $\gamma=0.7$.

flame propagation occurs for $L e_{F}<1$. However, for $L e_{F}>1$ the flame suffers from oscillatory instabilities. In these cases, both the linear stability analysis of the symmetric base solution and the unsteady computations were used to predict the Hopf bifurcation points.

\section{Scale of the problem and thermodynamic properties}

The dimensionless form of the governing equations can conceal the magnitude of the physical size of the problem. To estimate the order of magnitude of the channel height together with the thermodynamic and transport properties of the fuel-oxidiser mixtures, a series of detailed chemistry computations of freely-propagating planar premixed flames was performed with the code LFLAM, which is based on the equations described in [44].

Since the present work investigates the influence of $L e_{F}$ and $L e_{Z}$ on the dynamics of the flame in microchannels, three different mixtures are selected corresponding to different values of the fuel diffusivity. For example, for $L e_{F}<1$ we choose a lean hydrogen-air mixture at equivalence ratio $\phi=0.5$, such as done in [17-19]. For $L e_{F}=1$ a lean methaneair mixture at $\phi=0.6$ is selected. Finally, for $L e_{F}>1$ a lean mixture at $\phi=0.5$ of butane diluted in a $21 \%$ oxygen-helium environment is used, as experimentally investigated in the dynamics of freely-propagating flames [45], where a value of $L e_{F} \approx 4$ in the unburnt mixture was reported. It is seen that the dilution of fuels with light species results in a significant increase of the value of the fuel Lewis number.

The detailed kinetic mechanism used during the planar flame computations was the well-known San Diego mechanism [46] for hydrogen-air and methane-air mixtures, and that proposed in [47] and available at [48] for butane-oxygen-helium mixtures. Note that the laminar flame speed computed here was slightly smaller than that reported in [17] for the hydrogen-air mixture at the same conditions $\left(S_{L}=58 \mathrm{~cm} / \mathrm{s}\right)$, probably because thermal diffusion was excluded in those calculations. 
Table 1. Thermodynamics and transport properties for three different mixtures at pressure $p=$ $1 \mathrm{~atm}$ and $T_{0}=300 \mathrm{~K}$. The estimated value of the channel height is $h$.

\begin{tabular}{lcccccc}
\hline Mixture & $\phi$ & $S_{L}(\mathrm{~cm} / \mathrm{s})$ & $\mathcal{D}_{T}\left(\mathrm{~m}^{2} / \mathrm{s}\right)$ & $L e_{F}$ & $L e_{\mathrm{H}}$ & $h(\mathrm{~mm})$ \\
\hline $\mathrm{H}_{2}$-air & 0.5 & 48.7 & $3.42 \times 10^{-5}$ & 0.3 & 0.25 & 0.3 \\
$\mathrm{CH}_{4}$-air & 0.6 & 10.4 & $2.24 \times 10^{-5}$ & 1.0 & 0.17 & 1.0 \\
$\mathrm{C}_{4} \mathrm{H}_{10}-\mathrm{O}_{2}-\mathrm{He}$ & 0.5 & 54.0 & $2.78 \times 10^{-5}$ & 3.9 & 0.42 & 0.2 \\
\hline
\end{tabular}

In our calculations, we kept the value of the Damköhler number fixed at $d=20$. As the value of the channel height for each reactive mixture can be determined from the dimensionless parameters presented above, according to $h=\left(\mathcal{D}_{T} \sqrt{d}\right) / S_{L}$, Table 1 shows the values of $h$ obtained for the three different mixtures. They correspond to micro scale in all cases. The Lewis numbers of the fuel and $\mathrm{H}$-radical were estimated from multicomponent mixture properties in the reactive-diffusive zone of the flame and they compare with the values of $L e_{F}$ used in the present work. The Lewis numbers increase slightly towards the unburnt mixture. For example, a value of $L e_{F}=0.35$ was estimated in the fresh zone for the hydrogen-air mixture. The value of $\mathcal{D}_{T}$ was estimated in the fresh gas mixture.

\section{The linear stability analysis}

The linear stability analysis of the symmetric steady flame is formulated introducing a small harmonic perturbation in the form

$$
\begin{aligned}
& \theta(x, y ; t)=\theta_{0}(x, y)+\epsilon \theta_{1}(x, y) \exp (\lambda t), \\
& F(x, y ; t)=F_{0}(x, y)+\epsilon F_{1}(x, y) \exp (\lambda t), \\
& Z(x, y ; t)=Z_{0}(x, y)+\epsilon Z_{1}(x, y) \exp (\lambda t),
\end{aligned}
$$

where $\lambda \in \mathbb{C}$, the subindex 0 denotes the base solution obtained in the symmetric steadystate calculations and $\epsilon$ is a small perturbation. As clarified in [6], it is not necessary to introduce perturbations of $u_{f}$ in the analysis, and therefore the linearised eigenvalue problem obtained when substituting the perturbation (15) into Equations (6)-(11) leads directly to the following two-dimensional homogeneous problem:

$$
\begin{aligned}
\lambda \theta_{1}=- & \sqrt{d}\left\{u_{f}+6 m y(1-y)\right\} \frac{\partial \theta_{1}}{\partial x}+\Delta \theta_{1}+d \mu q Z_{1}, \\
\lambda F_{1}= & -\sqrt{d}\left\{u_{f}+6 m y(1-y)\right\} \frac{\partial F_{1}}{\partial x}+\frac{1}{L e_{F}} \Delta F_{1} \\
& -d \mu k\left(\theta_{0}\right)\left\{A \theta_{1}+Z_{0} F_{1}+F_{0} Z_{1}\right\}, \\
\lambda Z_{1}= & -\sqrt{d}\left\{u_{f}+6 m y(1-y)\right\} \frac{\partial Z_{1}}{\partial x}+\frac{1}{L e_{Z}} \Delta Z_{1} \\
& +d \mu k\left(\theta_{0}\right)\left\{A \theta_{1}+Z_{0} F_{1}+F_{0} Z_{1}\right\}-d \mu Z_{1},
\end{aligned}
$$

where $A=\frac{\beta}{\left[1+\gamma\left(\theta_{0}-1\right)\right]^{2}} Z_{0} F_{0}$. 
To study the stability of the symmetric steady flames, Equations (16)-(18) should be considered in half of the domain with the corresponding boundary conditions

$$
\begin{gathered}
x \rightarrow-\infty: \quad \theta_{1}=F_{1}=Z_{1}=0, \\
x \rightarrow+\infty: \quad \partial \theta_{1} / \partial x=\partial F_{1} / \partial x=\partial Z_{1} / \partial x=0, \\
y=0: \quad \partial \theta_{1} / \partial y=\partial F_{1} / \partial y=\partial Z_{1} / \partial x=0,
\end{gathered}
$$

and two kinds of boundary conditions in the midplane $y=1 / 2$ which select either the symmetric mode: $\partial \theta_{1} / \partial y=\partial F_{1} / \partial y=\partial Z_{1} / \partial y=0$, or the non-symmetric mode: $\theta_{1}=F_{1}=$ $Z_{1}=0$, of the perturbation.

Since the main goal of the linear stability analysis is to determine whether a given steady solution is stable or not, it is enough to have information only about the eigenvalue with the largest real part. For this reason, the present work uses the simple time-dependent method developed in [6] for calculating the main eigenvalue. This method is simple to implement and has a numerical cost comparable to that of calculating a steady solution using a time-marching procedure. If the real part of the main eigenvalue obtained is positive, $\mathfrak{R}(\lambda)=\lambda_{R}>0$, then the steady symmetric solution is unstable, and conversely, if $\lambda_{R}<0$ the solution is linearly stable. If the imaginary part is also positive, $\Im(\lambda)>0$, the oscillatory instability mode is excited. The time-dependent method requires some initial conditions. These were choosen in the form

$$
\begin{aligned}
& \theta_{1}=K_{1} \exp \left\{-\left[\left(x-x_{0}\right)^{2}+\left(y-y_{0}\right)^{2}\right] / r_{0}^{2}\right\}, \\
& F_{1}=K_{2} \exp \left\{-\left[\left(x-x_{0}\right)^{2}+\left(y-y_{0}\right)^{2}\right] / r_{0}^{2}\right\}, \\
& Z_{1}=K_{3} \exp \left\{-\left[\left(x-x_{0}\right)^{2}+\left(y-y_{0}\right)^{2}\right] / r_{0}^{2}\right\},
\end{aligned}
$$

where $K_{1}, K_{2}$ and $K_{3}$ are non-zero complex constants with the corresponding real and imaginary part of order unity and $r_{0}$ is the characteristic radius. The values $K_{1}=1-i$, $K_{2}=1-i, K_{3}=1+i, y_{0}=0.3$, and $r_{0}=0.1$ were used typically. The value $i$ represents the imaginary part. The reference point $x_{0}$ was selected at a small distance upstream of the region where $Z_{0}$ peaks.

When the above method is applied to the analysis of the symmetric modes, it always gives the eigenvalue $\lambda=0$ in cases when the symmetric mode is stable. As clearly explained in [6], the steady solutions of the travelling waves are always invariant with respect to a shift along the direction of motion and therefore it always leads to the existence of $\lambda=0$ with the eigenfunctions given by $\left(\theta_{1}, F_{1}, Z_{1}\right)=\left(\partial \theta_{0} / \partial x, \partial F_{0} / \partial x, \partial Z_{0} / \partial x\right)$ because of the applied Neumann boundary conditions.

\section{Numerical treatment}

As explained above, steady-state and time-dependent computations, together with linear stability analysis, were carried out in a finite domain, $x_{\min } \leq x \leq x_{\max }$. Typical values were $x_{\min }=-10$ and $x_{\max }=10$, but they were varied to ensure independence of the results for each case. The condition $\Delta x^{\prime} \ll \delta_{T}$ was selected to achieve enough spatial resolution in the thin flame zone where the intermediate species exist, that is, $\Delta x \ll d^{-1 / 2}$. For the value $d=20$ used in the calculations below, $\Delta x \ll 0.2$. The spatial derivatives were discretised using second-order, three-point central differences on a rectangular uniform grid. Typical 
numbers of points were $2001 \times 101$ for the $x$ - and $y$-coordinates, respectively, ensuring the condition above, $\Delta x=\Delta y=0.01 \ll 0.2$. The number of points was doubled in some cases to test grid independence.

The steady-state problem was solved using a simple Gauss-Seidel iterative method with over-relaxation. The time-dependent calculations were carried out with a simple explicit time-marching procedure with first-order discretisation in time. Due to the nonlinearity of the reaction rate terms, specially important in the chain-branching description, a sufficiently small time step $\Delta t$ was necessary. The typical value was $\Delta t=10^{-6}$. However, the value of the time step was fixed to $\Delta t=10^{-7}$ during the time-dependent calculations of the stability problem; see details of the method in [6].

Computations were carried out in a Linux cluster using 16 processors running with a clockspeed of $2.93 \mathrm{GHz}$. Steady calculations required between 0.1 and $0.5 \mathrm{~h}$. Unsteady and stability calculations could take up to $12 \mathrm{~h}$.

\section{Results}

Figures $3-5$ show the variation of the steady flame propagation velocity with the flow rate for values of the heat of reaction $q=2,1.5$ and 1.2, respectively. The smaller values of $q$ correspond to flames near the flammability limit. In Figure 5, we also show a particular case very close to the flammability limit, namely the case $q=0.9$ and $L e_{F}=4$, chosen to study the oscillatory instability, as explained below in Section 6.2. Since the mass fraction of fuel is included in the dimensionless form of the heat of reaction, $q$ plays here the role of the equivalence ratio for sufficiently fuel-deficient mixtures, so by using this simple chainbranching model the influence of the equivalence ratio on the stability of the steady-state flames can be addressed.

In the figures, solid curves represent steady and stable solutions (symmetric or nonsymmetric flames) and dashed ones indicate unstable symmetric solutions, obtained by reducing the domain to half of its height. These symmetric solutions are used in the linear stability analysis. The influence of $L e_{F}$ and $L e_{Z}$ on the propagation velocity is shown below. Although the value of $L e_{Z}$ is usually smaller than $L e_{F}$ in typical combustion processes, herein we study independently all ranges of values for completeness. We distinguish two cases: the first one corresponding to $L e_{F}<1$, where only steady flame propagation emerges, and the second one to $L e_{F}>1$, when unsteady flame dynamics appear.

\subsection{Symmetry-breaking bifurcation for $\mathrm{Le}_{\mathrm{F}}<1$}

For $L e_{F}<1$ the flame propagates steadily as a whole. A multiplicity of solutions can exist - both symmetric and non-symmetric flame solutions.

Let us focus on Figure 3(a). For sufficiently negative values of $m$ (assisted flow) the flame shape is always symmetric and propagates upstream $\left(u_{f}>0\right)$. When $m$ is increased to sufficient positive values (opposed flow) the flame switches to a non-symmetric flame shape, only for $L e_{F}<1$. This symmetry-breaking bifurcation occurs when $m=m_{b}$, where $m_{b}$ is the largest flow rate value with $S=0$. In the figures, the symbol $\bullet$ marks the bifurcation point. Solid curves for $m<m_{b}$ represent stable symmetric solutions and solid curves for $m>m_{b}$ indicate stable non-symmetric ones. The dashed curves for $m>m_{b}$ corresponds to unstable symmetric solutions, as commented before.

The flashback point, defined as the critial flow rate for which the flame propagates upstream, is marked with the symbol $\circ$ in the figures. Flames of lighter mixtures such 

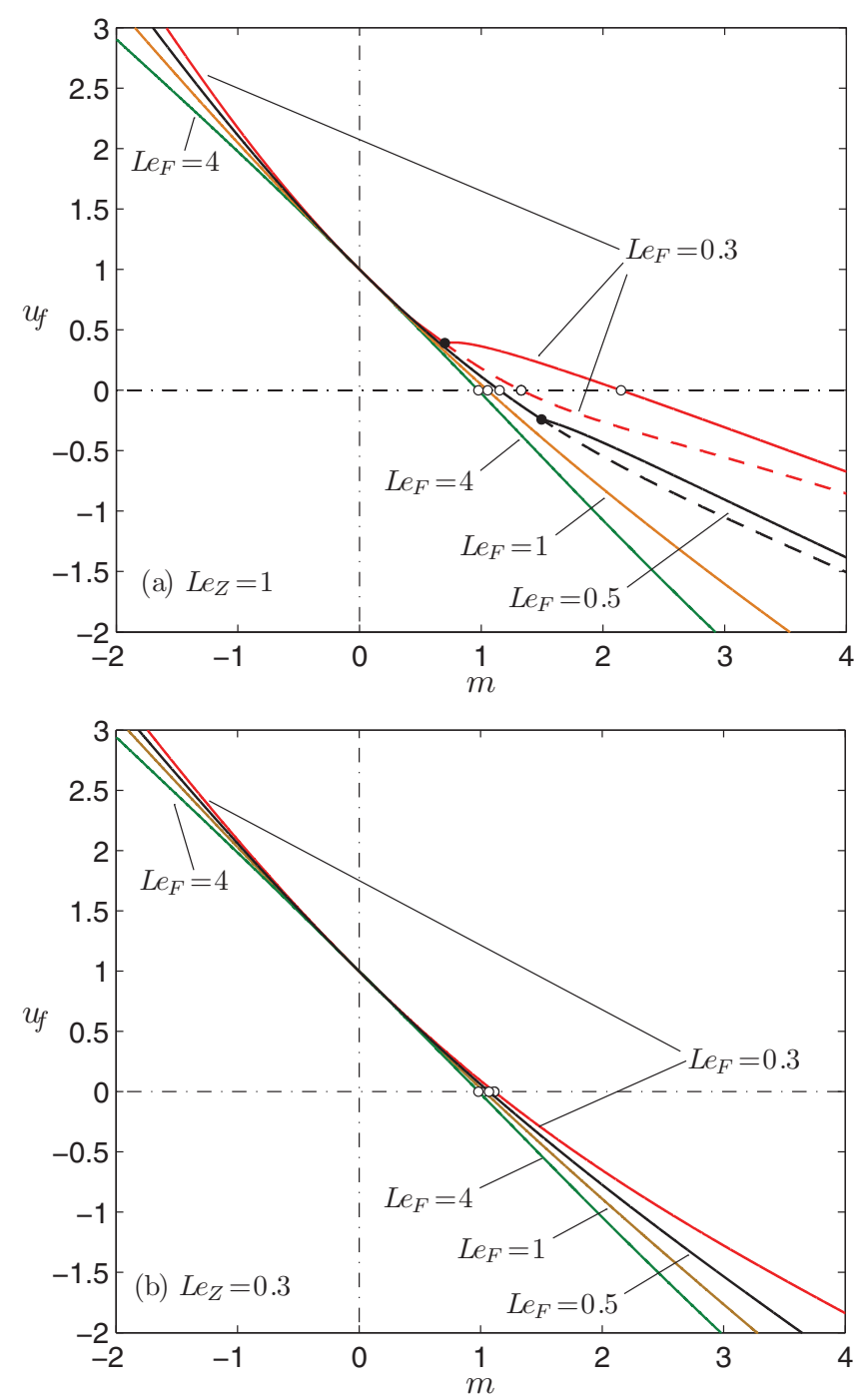

Figure 3. The variation with the flow rate of the propagation velocity of the flame for four different fuel Lewis numbers. Calculated for $d=20, q=2$ and: (a) $L e_{Z}=1$; (b) $L e_{Z}=0.3$. Solid curves indicate stable and steady solutions (symmetric flame shape for $m<m_{b}$ and non-symmetric for $m>$ $m_{b}$ ) and dashed curves represent unstable symmetric solutions.

as $L e_{F}=0.3$ can propagate upstream with larger values of the opposed flow rate than heavier mixtures, such as $L e_{F}=1$, for example. This effect is associated with an increase of the temperature due to flame curvature and diffusion effect, which also becomes more important for flames close to the flammability limit. However, if the intermediate species are very diffusive, as in the case with $L e_{Z}=0.3$ shown in Figure 3(b), its concentration in the curved flame is sunk by diffusion, in particular in the transversal direction, then promoting the symmetry of the flame. Figure 3(b) shows the flame velocity with the flow rate, but only symmetric solutions were found. The high diffusion of $Z$ stabilises the flame to symmetric shapes in all cases. 

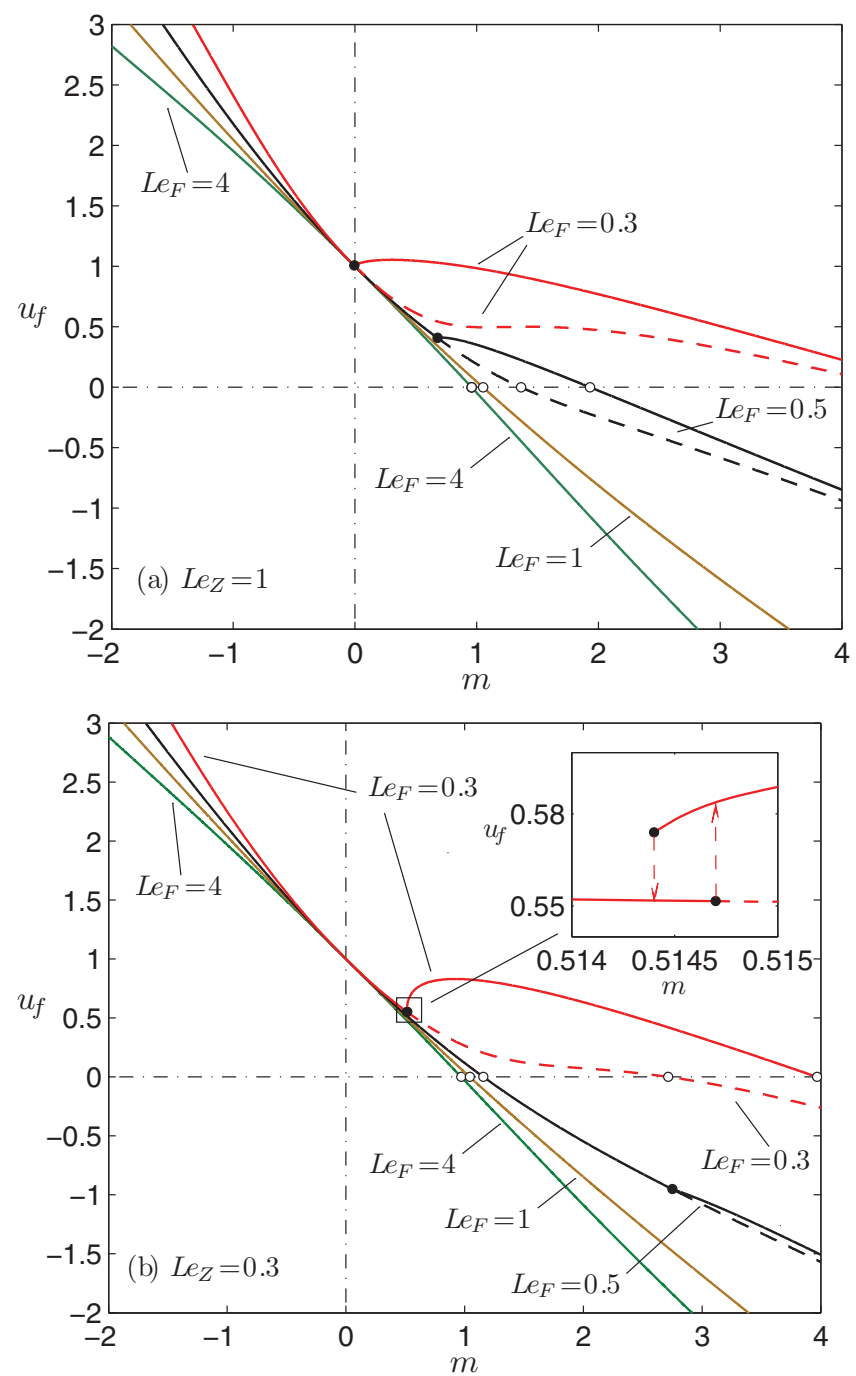

Figure 4. The variation with the flow rate of the propagation velocity of the flame for four different fuel Lewis numbers. Calculated for $d=20, q=1.5$ and: (a) $L e_{Z}=1$; (b) $L e_{Z}=0.3$. The inset in (b) shows the multiplicity of solutions that emerge for $L e_{F}=0.3$.

A similar effect is found for $q=1.5$ and $q=1.2$ in Figures 4 and 5, respectively. As the flame becomes leaner, by reducing the value of $q$, symmetry-breaking bifurcation is anticipated to smaller values of $m$, but the effect of $L e_{Z}$ decrease in importance.

Most of the bifurcations found were supercritical, that is, a stable non-symmetric branch and a non-stable symmetric one emerge from a stable symmetric solution. However, subcritical bifurcations arise when reducing the value of $q$. This is shown in the inset in Figure 4(b), where multiplicity of stable solutions was found for $L e_{F}=0.3$. To clarify this point better, Figure 6 shows the bifurcation diagram in the $S-m$ plane for this case, i.e. $L e_{F}=0.3$ and $q=1.5$. Three stable solutions become possible for the same value of $m$ : one symmetric $(S=0)$ and two non-symmetric $(S<0$ or $S>0)$ when reducing the value of 

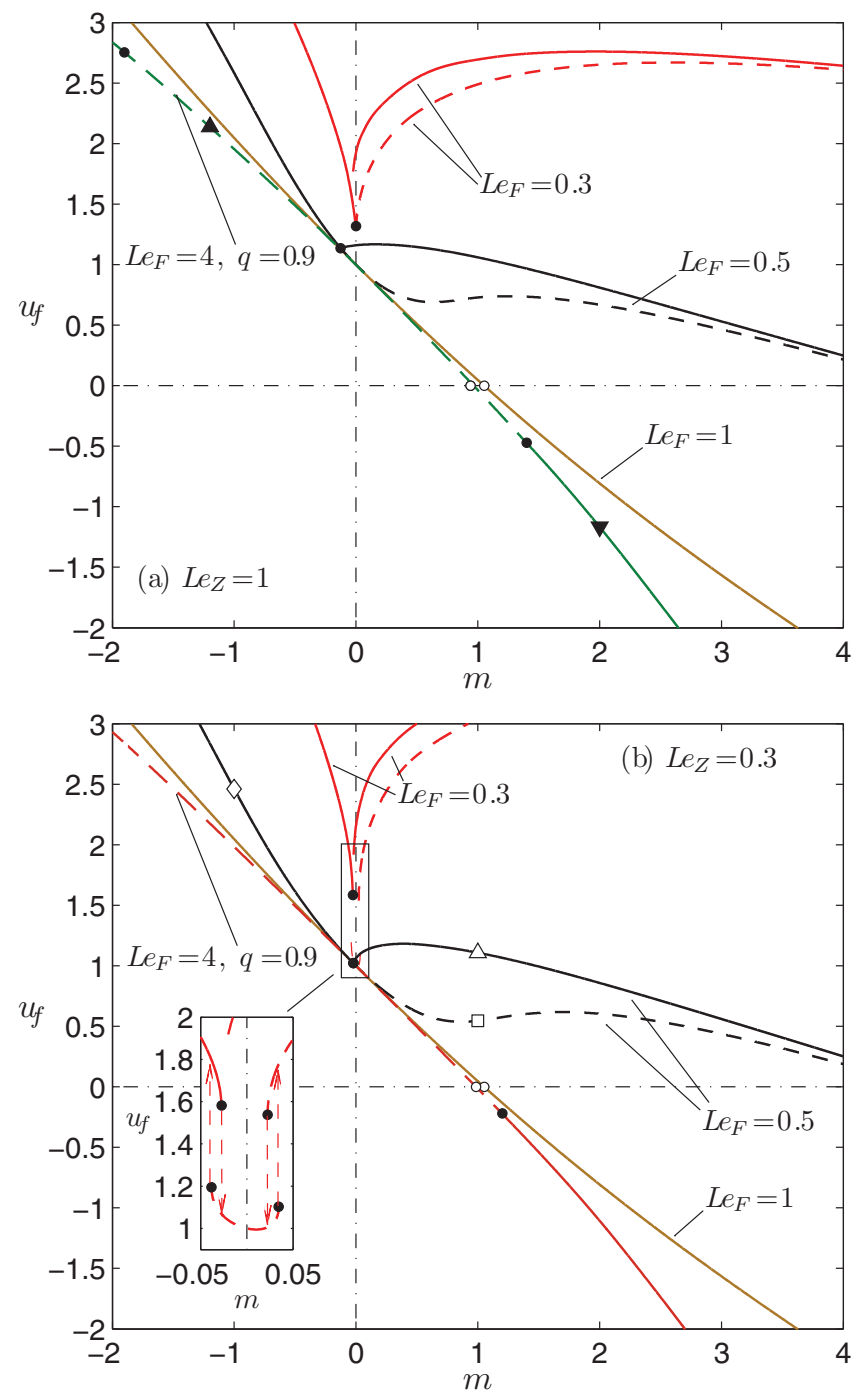

Figure 5. The variation with the flow rate of the propagation velocity of the flame for four different fuel Lewis numbers. Calculated for $d=20, q=1.2$ and: (a) $L e_{Z}=1$; (b) $L e_{Z}=0.3$, except in the case $L e_{F}=4$, which was calculated for $q=0.9$. In this case in (a), the symbols $\boldsymbol{\Delta}$ and $\boldsymbol{\nabla}$ show the samples plotted in Figures 9 and 11 for a time-dependent calculation, respectively. The symbols $\diamond$, $\square$ and $\triangle$ in (b) correspond to the flame structure plotted in Figure 7. The inset in (b) shows more clearly the intricate hysteresis for $L e_{F}=0.3$.

$L e_{z}$. The connective branches between the bifurcation point and the non-symmetric solutions are unstable and are not plotted in the figures. Hysteresis showing a double solution has already been reported in the dynamics of hydrogen-air flames in micro- and meso-scale channels with a prescribed wall temperature $[17,18]$. The inset in Figure 4(b) shows the same effect, that is, hysteresis in the symmetric solution branch.

For fuel-lean flames, such as $q=1.2$ in Figure 5, the influence of the diffusivity of radicals in the flame stabilisation to symmetric solutions becomes almost negligible due to 


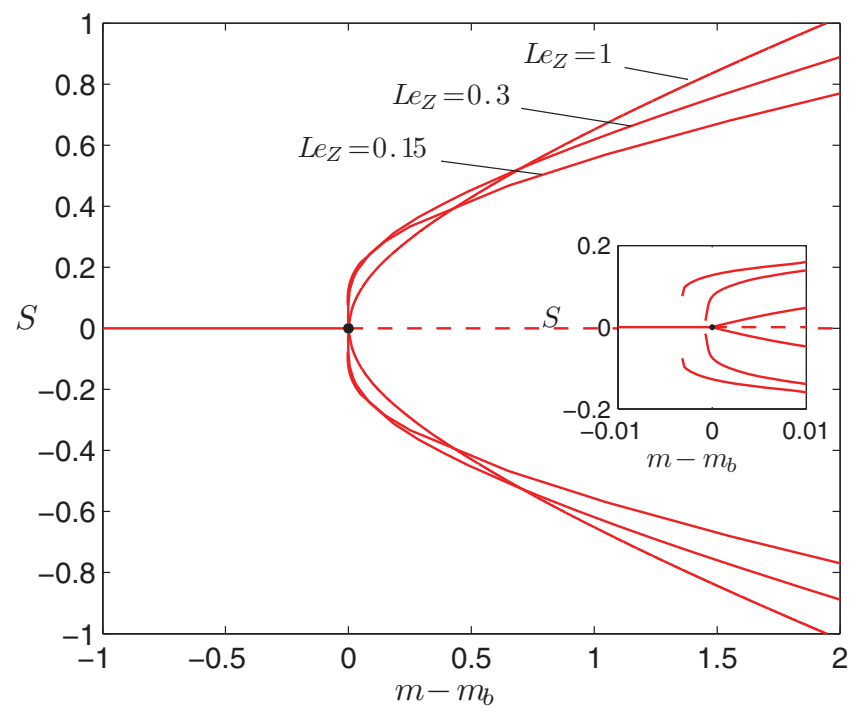

Figure 6. Bifurcation diagram representing the parameter $S$ as a function of the relative flow rate $m-m_{b}$ for three different $L e_{Z}$. Calculated for $d=20, q=1.5$ and $L e_{F}=0.3$. The critical values $m_{b}=-0.006,0.5146$ and 0.9499 correspond to the flow rate at the bifurcation point for $L e_{Z}=1$, $L e_{Z}=0.3$ and $L e_{Z}=0.15$, respectively. The inset blows up the near bifurcation region showing that three stable solutions are possible for subcritical bifurcations.

the small radicals concentrations found close to the flammability limit. However, for $m=$ 0 , we can still see the stabilising effect of the radical diffusion. For example, Figure 5(a) shows flames that propagate faster than that of the planar one for $L e_{F}=0.5$ and $L e_{F}=0.3$. This indicates the existence of cellular structures that emerge for very diffusive fuels and sufficiently lean flames. In Figure 5(b), where we decrease the value of $L e_{Z}$ to 0.3 , the cellular structure disappears when $L e_{F}=0.5$ and the flame becomes planar again, propagating at $u_{f}=1$. It is interesting to comment here briefly on the effect of the Damköhler number. When the characteristic size of the cellular flame is comparable with that of the channel height, this cellular flame emerges. Larger values of $d$ allow the existence of cellular structures for larger values of $L e_{F}$. The same was demonstrated for symmetric flames in [6], that is, symmetric solutions become unstable to non-symmetric solutions for a sufficiently wide channel. The inset in Figure 5(b) shows the intricate hysteresis found in the symmetric solution for $L e_{F}=0.3$.

Figure 7 depicts the typical flame structure found in the computations. They correspond to flames obtained in the case $q=1.2, L e_{F}=0.5$, and $L e_{Z}=0.3$, marked with symbols in Figure 5(b). For $m<1$ the flame shows a mushroom-like shape and for $m>1$ the flame is tulip-shaped whenever it is stable, otherwise it shows a non-symmetric slant-like shape. These flame shapes in ducts were described before for a complete Navier-Stokes formulation [28,30], and a thorough explanation of the flame-flow interaction in tulip flames during the flame propagation in closed ducts was described in [49]. Note the increase of temperature above the adiabatic value (dashed lines) due to flame curvature. The maximum temperature is higher in the non-symmetric case.

The linear stability analysis of the symmetric flame solution helps to confirm the location of the bifurcation points. They are all in agreement with the results predicted by steady-state 

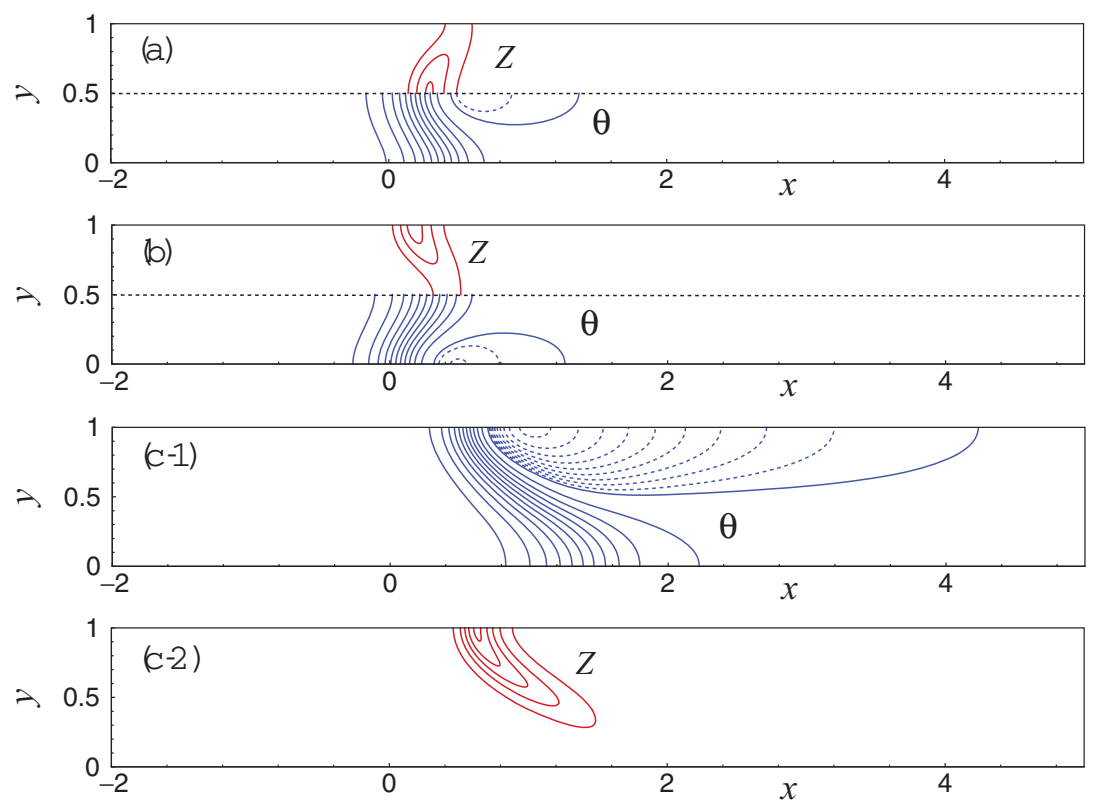

Figure 7. Flame structure for steady symmetric and non-symmetric flames. Calculated for $q=1.2$, $L e_{F}=0.5$ and $L e_{Z}=0.3$. Isotherms are plotted with solid lines at intervals of 0.1 up to $\theta=1.2$. Dashed lines correspond to isotherms from the adiabatic temperature $\theta=1.2$ at intervals of 0.02 . The contours of the radical mass fraction are plotted at intervals of 0.02. (a) Symmetric mushroom-shaped flame for $m=-1$. (b) Symmetric tulip-shaped flame for $m=1$. Non-symmetric slant-shaped flame for $m=1$ : (c-1) isotherms and (c-2) radical mass fraction contours. The flame structures (a), (b) and (c) correspond to the symbols $\diamond, \square$ and $\triangle$ in Figure 5(b), respectively.

and time-dependent calculations. Figure 8 shows the growth rate of the real part of the main eigenvalue $\lambda_{R}$ with the flow rate $m$ for a constant value of $q=1.5$ and for different values of $L e_{F}$ and $L e_{Z}$ as an example. The imaginary part is not plotted here because its value is always zero. All the curves correspond to the non-symmetric perturbative mode. The plot shows that the transition to a non-symmetric flame shape occurs for larger values of $m$ when $L e_{Z}$ is reduced. The effect of the differential diffusion of $Z$ is then to stabilise the symmetric flame shape, as commented above, although this effect becomes less important for smaller values of $L e_{F}$.

\subsection{Oscillatory instability for $\mathrm{Le}_{\mathrm{F}}>1$}

Oscillatory instability only emerges for sufficiently large values of $L e_{F}>1$ and small enough values of the heat of reaction $q$, that is, only for flames close to the flammability limit. As an example, the cases with $L e_{F}=4$ and $q=0.9$ are shown in Figure 5, where the the symbol $\bullet$ marks in this case a supercritical Hopf bifurcation indicating that the symmetric flame solution turns into an unstable and oscillatory mode (dashed line) with a non-symmetric shape fluctuating in time between the adiabatic walls. The solution again becomes steady and symmetric for sufficiently large values of the assisted or opposed flow rates (solid line).

In order to clarify the flame dynamics, Figure 9 shows the isocontours of the radical mass fraction plotted at different instants for the time-dependent computations. The figure 


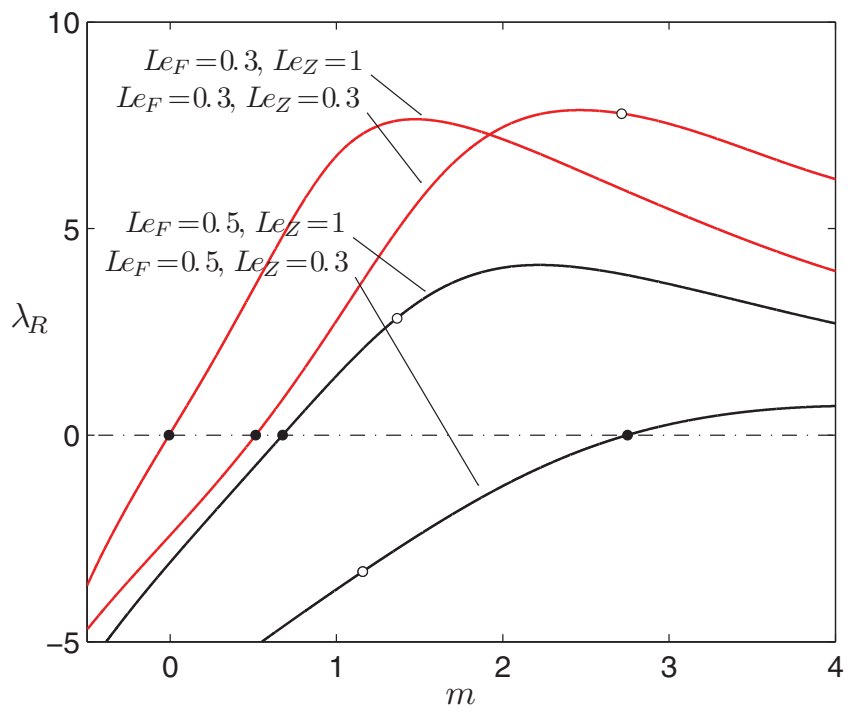

Figure 8. The growth rate of $\lambda_{R}$ as a function of the flow rate $m$ for $q=1.5$. The symbol corresponds to the bifurcation point and the symbol $\circ$ to the critical flashback point.

corresponds to the case $L e_{F}=4, L e_{Z}=1, q=0.9$ and assisted flow $m=-1.2$ plotted with the symbol $\boldsymbol{\Delta}$ in Figure 5(a). Note in Figure 9 that, because of the hot-spot initial conditions (12), another symmetric flame, not attached to the reference frame, propagates downstream; see the plot at $t=0.52$. Actually, this flame corresponds to the opposed flow
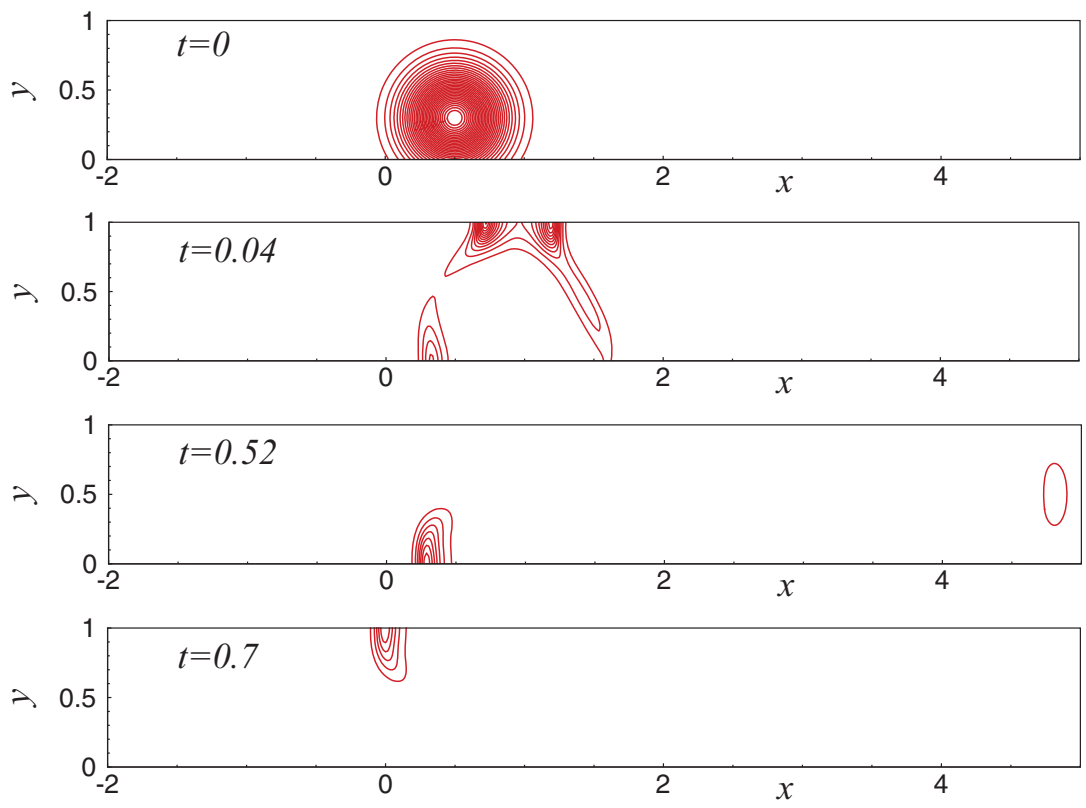

Figure 9. Evolution of the flame from the hot-spot initial condition (12) calculated for $L e_{F}=4$, $L e_{Z}=1, q=0.9$ and $m=-1.2$. The figure plots the isocontours of $Z$ (at intervals of 0.03 ) at four instances, some marked with the symbol $\circ$ in Figure 14. 


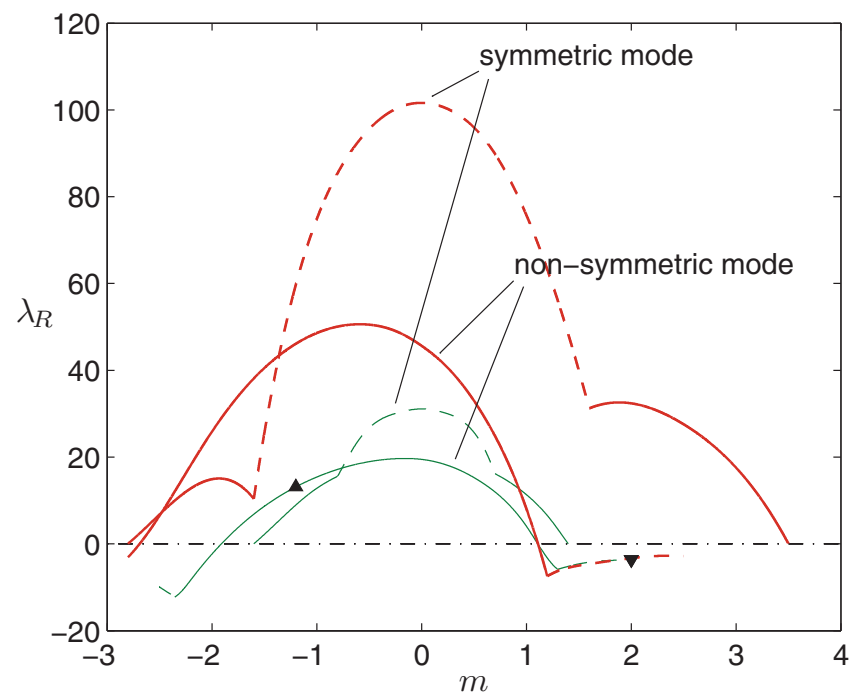

Figure 10. The growth rate $\lambda_{R}$ as a function of the flow rate $m$ for the case $L e_{F}=4$ and $q=0.9$. Thin curves correspond to $L e_{Z}=1$ and thick curves to $L e_{Z}=0.3$. The curves are plotted with solid lines when the imaginary part $\Im(\lambda) \neq 0$ and with dashed lines when $\Im(\lambda)=0$. The $Z$ isocontours for the cases $m=-1.2$ (symbol $\mathbf{\Delta}$ ) and $m=2$ (symbol $\mathbf{\nabla}$ ) are plotted in Figures 9 and 11, respectively.
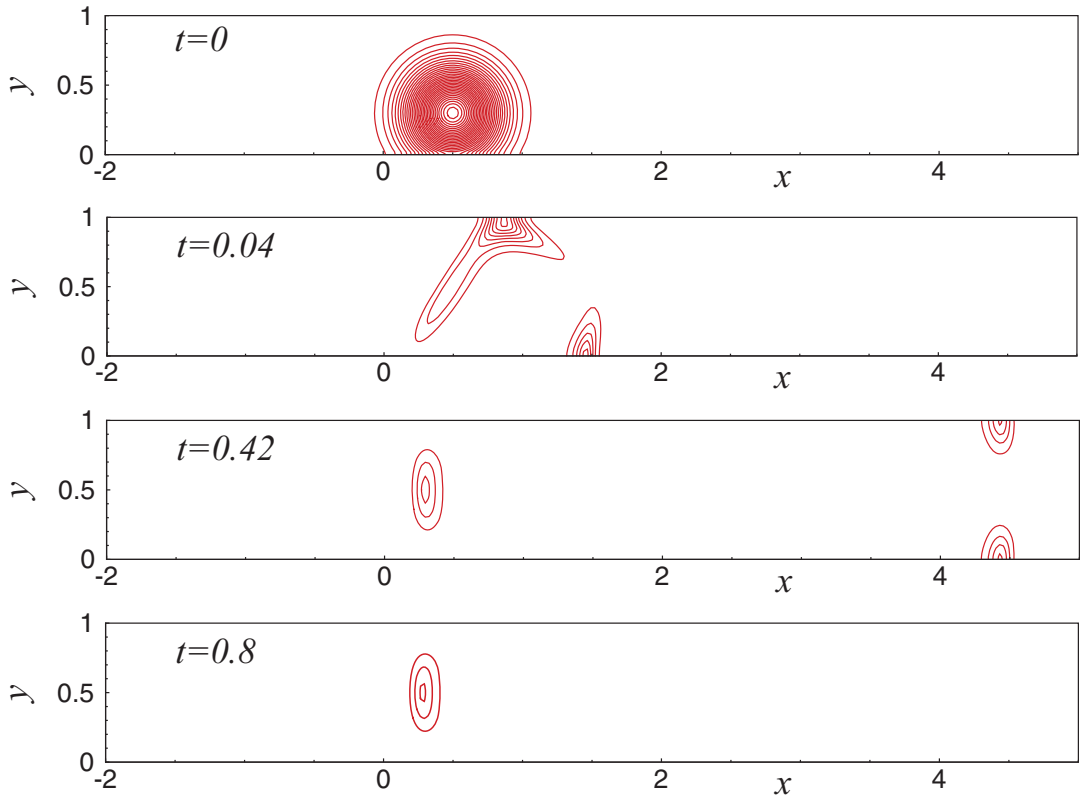

Figure 11. Evolution of the flame from the hot-spot initial condition (12) calculated for $L e_{F}=4$, $L e_{Z}=1, q=0.9$ and $m=2$. The figure plots the isocontours of $Z$ (at intervals of 0.03 ) at four instances, some marked with the symbol • in Figure 14. 


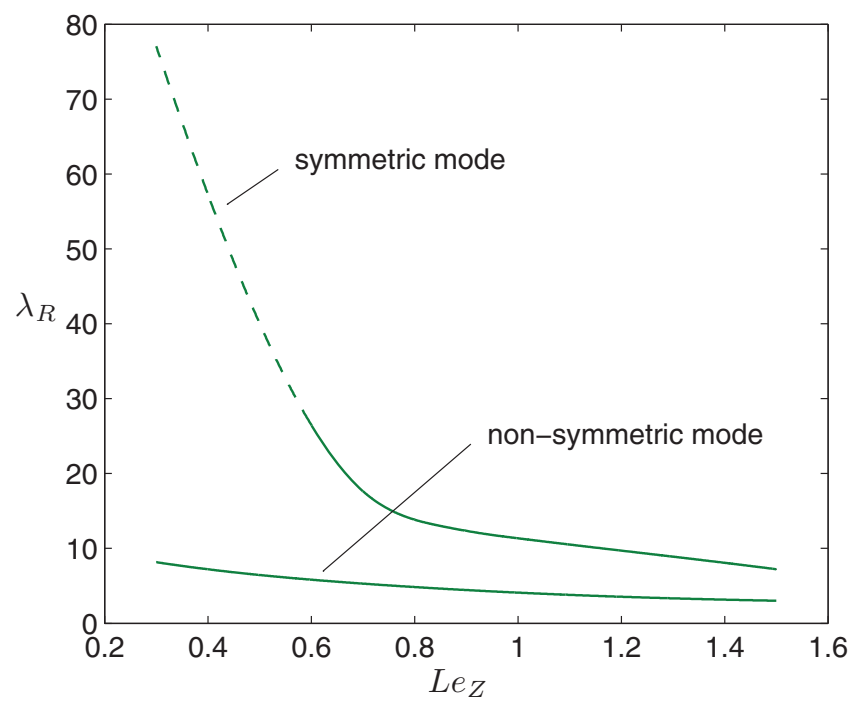

Figure 12. The growth rate $\lambda_{R}$ as a function of $L e_{Z}$ for $L e_{F}=4, q=0.9$ and $m=1$. The curves are plotted with solid lines when $\Im(\lambda) \neq 0$ and with dashed lines when $\Im(\lambda)=0$.

case $m=1.2$, and after a long computation it showed a symmetric oscillation at first but was finally extinguished. Animation 1, included in the supplementary material (see the section 'Supplemental data' immediately preceding the references list), clearly shows this behaviour. The evolution in time of the isocontours of $Z$ is plotted and the flame dynamics is better observed.

The linear stability analysis demonstrates that both flames, that is, the case $m=-1.2$ and $m=1.2$, become unstable $\left(\lambda_{R}>0\right)$, as depicted in Figure 10 with thin lines, and

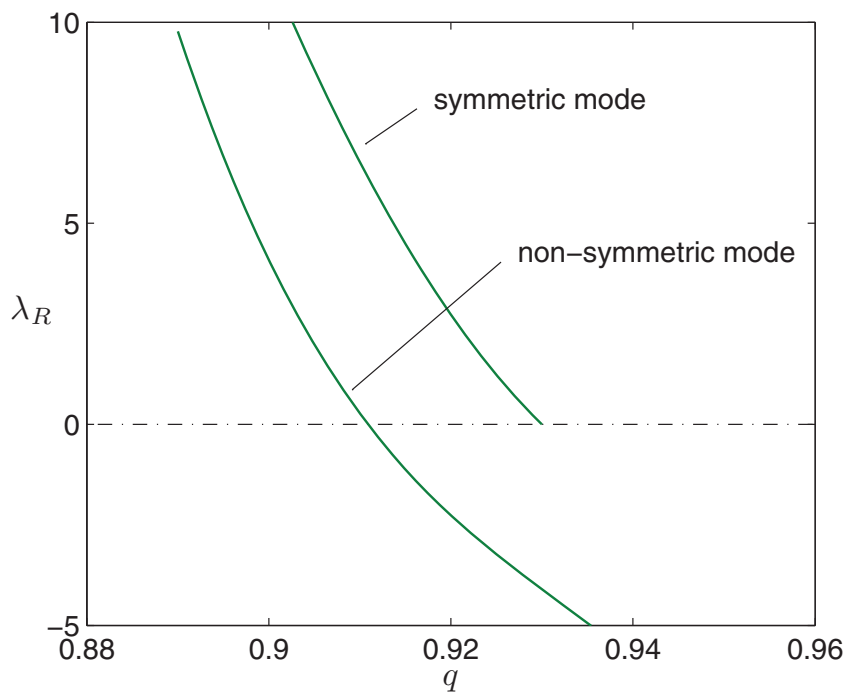

Figure 13. The growth rate $\lambda_{R}$ as a function of $q$ for $L e_{F}=4, L e_{Z}=1$ and $m=1$. The curves are plotted with solid lines when $\Im(\lambda) \neq 0$ and with dashed lines when $\Im(\lambda)=0$. 

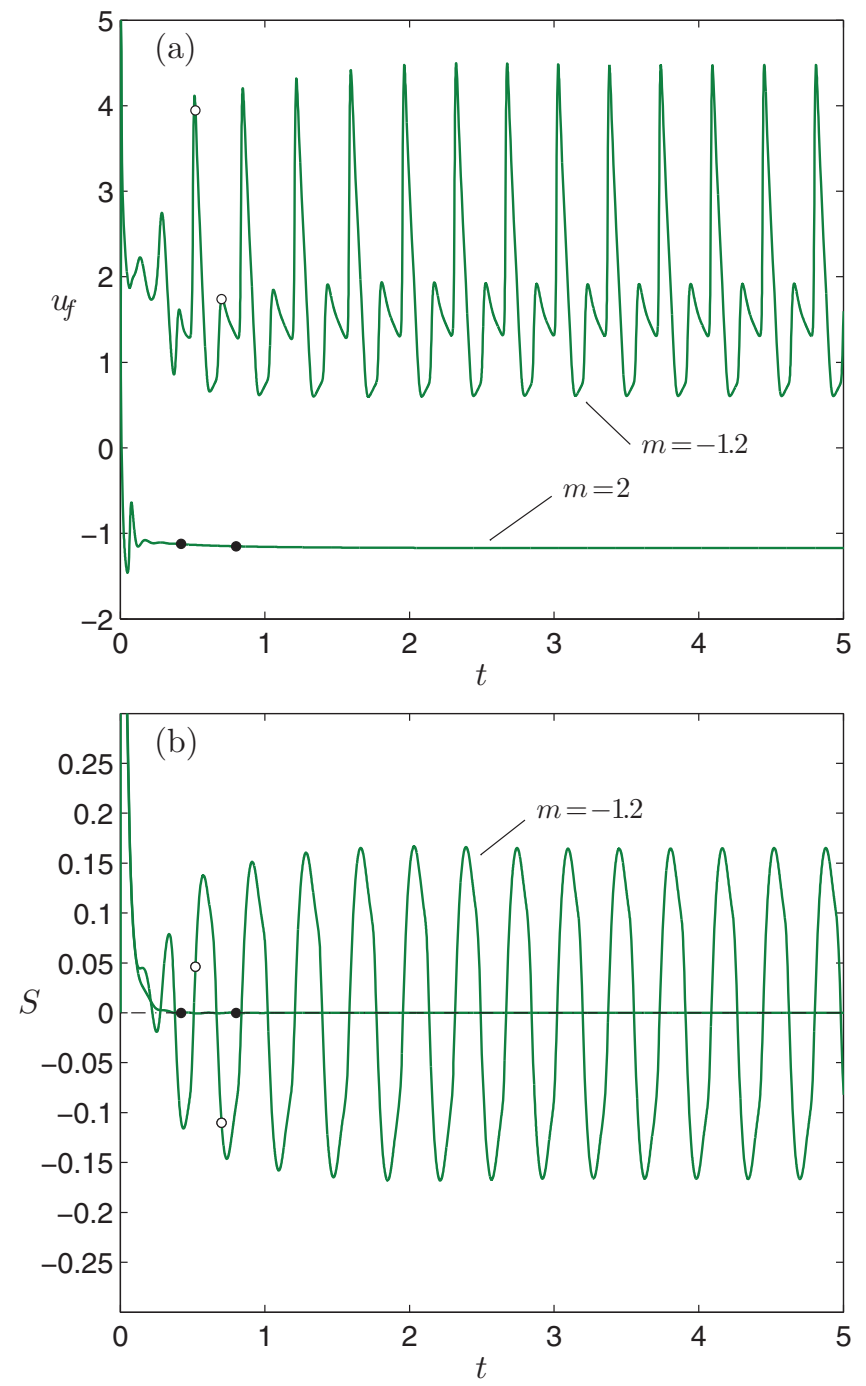

Figure 14. Time histories of (a) the flame propagation $u_{f}$ and (b) the symmetry indicator $S$, calculated for $L e_{F}=4, L e_{Z}=1, q=0.9$ and $m=-1.2$ and 2 .

with the symbol $\boldsymbol{\Delta}$ for $m=-1.2$. In this figure, solid lines are used when $\Im(\lambda)>0$. The non-symmetric oscillating mode is more prone to arise for $m=-1.2$. This was checked during time-dependent calculations. For $m=1.2$ the symmetric oscillating mode or pulsating mode would be more prone to emerge, but the flame extinguishes after some symmetric oscillations, as commented before. Other initial conditions were assumed during computations in order to check this extinction point, for example, by using the corresponding steady-state profiles perturbed with (12), but the flame always extinguished.

The same has been done for the case $L e_{F}=4, L e_{Z}=1, q=0.9$ and $m=2$, marked with the symbol $\mathbf{\nabla}$ in Figure 5(a). In this case, Figure 11 depicts the $Z$ isocontours for four instances, where the flame evolves to a steady flame solution. Also note that another flame propagates downstream at a constant velocity; see $t=0.42$. It corresponds to the case 
$m=-2$. Animation 2 shows the flame dynamics for this case, where the steady flames propagating upstream and downstream are much better seen. Figure 10 shows with thin curves that both steady symmetric solutions are stable $\left(\lambda_{R}<0\right)$. The symbol $\mathbf{\nabla}$ stand for $m=2$. The case $m=0$ also exhibits extinction. It seems to be a characteristic of the chain-branching kinetics, not seen in the one-step model, that the flame becomes unstable to extinction before reaching the theoretical flammability limit for $L e_{F}>1$ cases [42].

The role of the differential diffusion of the radical is completely reversed for $L e_{F}>$ 1 in comparison to $L e_{F}<1$. Lighter radicals destabilise the flame to oscillatory modes. This can be seen in Figure 10, where the unstable region is increased when reducing $L e_{Z}$ to 0.3 . This tendency is tested in Figure 12, where $\lambda_{R}$ is plotted as a function of $L e_{Z}$ for the values of $L e_{F}=4, q=0.9$ and $m=1$. The main eigenvalue increases when $L e_{Z}$ decreases. When the mixture becomes leaner the flame is also destabilised, as seen in Figure 13, where the variation of $\lambda_{R}$ with $q$ for the case $L e_{F}=4, L e_{Z}=1$ and $m=1$ is shown. Flames with $q>0.93$ become stable in this case, indicating that only equivalence ratios close to the flammability limit suffer from unstable modes. A similar behaviour has been reported recently when studying the influence of the equivalence ratio in the flame stability in channels with a prescribed wall temperature and making use of an Arrhenius chemistry [50].

In Figure 14 we plot the time histories of $u_{f}$ and $S$ for the cases with $L e_{F}=4, L e_{Z}=1$ and $q=0.9$ for $m=-1.2$ and $m=2$, using the initial condition (12) in a time-dependent calculation. The non-symmetric oscillatory flame shows a period doubling for $m=-1.2$, but for $m=2$ the flame is steady. The time-dependent calculation agrees well with the solution given by the linear stability analysis. Period-two and period-four solutions have been reported earlier in the chain-branching kinetics of planar flame fronts [42].

It is interesting to note that the steady flame shape is also reversed for $L e_{F}>1$ in comparison to $L e_{F}<1$. For example, Figure 7(a) shows that mushroom-like shapes arise for assisted flows when $L e_{F}<1$; however, tulip-like shapes appear when $L e_{F}>1$ - see the flame shape that propagates downstream $(m=-2)$ at $t=0.42$ in Figure 11. The differential diffusion of the fuel modifies the characteristic flame shape. The same is true for opposed flows.

\section{Conclusions}

The role of the intermediate species in the stability of flames propagating in adiabatic and non-catalytic channels was addressed in the present paper, making use of a simple two-step chain-branching kinetics. In order to concentrate on the preferential diffusion of the species, the thermo-diffusive model was employed. The size of the channel was kept fixed to a micro scale $(h \approx 1 \mathrm{~mm}$ or $d=20)$ for selected values of $L e_{F}$ and $L e_{Z}$.

Calculations were carried out using a steady-state code, a time-dependent code and a linear stability analysis of the steady symmetric base solution. The results obtained with the different approaches were all in agreement.

For $L e_{F}<1$ the flame is always steady, but symmetry-breaking bifurcation arises at sufficiently large values of the opposed flow, that is, the symmetric solution breaks to a non-symmetric one. The assisted flow does not suffer from this symmetry-breaking effect, at least in the range of values studied here. It is seen that small values of $L e_{F}$, for instance $L e_{F}=0.3$, increase the flame curvature and the maximum temperature in this region. This effect allows the flame to remain in the channel at higher flow rates before flashback occurrence. However, small values of $L e_{Z}$ stabilise the flame again to symmetric solutions. This result is negative in the sense that the flame can no longer be held in the channel before 
flashback. For very lean mixtures, the role of $L e_{Z}$ was found to be less important due to the small radical concentration found in the reaction zone and cellular flame structures were identified in this regime.

For $L e_{F}>1$, oscillatory instabilities emerge only close to the flammability limit and for high enough values of $L e_{F}$. The Poiseuille flow stabilises the flame and the role of the intermediate species is opposite to the case with $L e_{F}<1$. Very diffusive radicals further destabilise the flame to oscillatory, pulsating or extinction modes.

The design of stable and practical microcombustors through catalytic reactions can make good use of the systematic study of the intermediate species involved in the chemical reactions and a theoretical simple chain-branching model allows first conclusions, as shown in the present work.

\section{Acknowledgements}

The authors would like to thank Dr Naud for his help in the detailed chemistry calculations of the thermodynamics and transport properties for the planar premixed flames with the use of the code LFLAM developed at CIEMAT.

\section{Funding}

This research was supported by the Spanish Ministerio de Ciencia e Innovación (MICINN) [Projects \#ENE2011-27686-C02-01, \#ENE2012-33213]; the Comunidad de Madrid [Project \#S2009/ENE1597, CONSOLIDER CSD2010-00011].

\section{Supplemental data}

The following supplemental data for this article can be accessed at http://dx.doi. org/10.1080/17509653.2014.946970.

Animation 1: Time evolution of the isocontours of $Z$ for the case $L e_{F}=4, L e_{Z}=1$, $q=0.9$, and $m=-1.2$. This corresponds to the case marked with the symbol $\Delta$ in Figure 10 .

Animation 2: Time evolution of the isocontours of $Z$ for the case $L e_{F}=4, L e_{Z}=1$, $q=0.9$, and $m=2$. This corresponds to the case marked with the symbol $\boldsymbol{\nabla}$ in Figure 10 .

\section{References}

[1] G.J. Sharpe, S.A.E.G. Falle, and J. Billingham, Numerical solutions of a model for the propagation of a surface-catalysed flame in a tube, IMA J. Appl. Math. 73 (2007), 107-122.

[2] Y. Ju and K. Maruta, Microscale combustion: Technology development and fundamental research, Prog. Energ. Combust. Sci. 37 (2011), 669-715.

[3] R. Chein, Y. Chen, and J.M. Chung, Numerical study of methanol-steam reforming and methanol-air catalytic combustion in annulus reactors for hydrogen production, Appl. Energy 102 (2013), 1022-1034.

[4] J. Daou and M. Matalon, Flame propagation in Poisenille flow under adiabatic conditions, Combust. Flame 124 (2001), 337-349.

[5] J. Daou and M. Matalon, Influence of conductive heat-losses on the propagation of premixed flames in channels, Combust. Flame 128 (2002), 321-339.

[6] V.N. Kurdyumov, Lewis number effect on the propagation of premixed flames in narrow adiabatic channels: Symmetric and non-symmetric flames and their linear stability analysis, Combust. Flame 158 (2011), 1307-1317.

[7] C. Cui, M. Matalon, J. Daou, and J. Dold, Effects of differential diffusion on thin and thick flames propagating in channels, Combust. Theory Model. 8 (2004), 41-64.

[8] V.N. Kurdyumov and E. Fernández-Tarrazo, Lewis number effect on the propagation of premixed laminar flames in narrow open ducts, Combust. Flame 128 (2002), 382-394. 
[9] V.N. Kurdyumov, G. Pizza, C.E. Frouzakis, and J. Mantzaras, Dynamics of premixed flames in a narrow channel with a step-wise wall temperature, Combust. Flame 156 (2009), 2190-2200.

[10] V.N. Kurdyumov and M. Matalon, Analysis of an idealized heat-recirculating microcombustor, Proc. Combust. Inst. 33 (2011), 3275-3284.

[11] M. Sánchez-Sanz, Premixed flame extinction in narrow channels with and without heat recirculation, Combust. Flame 159 (2012), 3158-3167.

[12] G.I. Sivashinsky, Diffusional-thermal theory of cellular flames, Combust. Sci. Technol. 15 (1977), 137-146.

[13] G. Joulin and G.I. Sivashinsky, Influence of momentum and heat losses on the large-scale stability of quasi-2D premixed flames, Combust. Sci. Technol. 98 (1994), 11-23.

[14] S.H. Kang, S.W. Baek, and H.G. Im, Effects of heat and momentum losses on the stability of premixed flames in a narrow channel, Combust. Theory Model. 10 (2006), 659-681.

[15] T.L. Jackson, J. Buckmaster, Z. Lu, D.C. Kyritsis, and L. Massa, Flames in narrow circular tubes, Proc. Combust. Inst. 31 (2007), 955-962.

[16] V.N. Kurdyumov and C. Jiménez, Propagation of symmetric and non-symmetric premixed flames in narrow channels: Influence of conductive heat-losses, Combust. Flame. 161 (2014), 927-936.

[17] G. Pizza, C.E. Frouzakis, J. Mantzaras, A.G. Tomboulides, and K. Boulouchos, Dynamics of premixed hydrogen/air flames in microchannels, Combust. Flame 152 (2008), 433-450.

[18] G. Pizza, C.E. Frouzakis, J. Mantzaras, A.G. Tomboulides, and K. Boulouchos, Dynamics of premixed hydrogen/air flames in mesoscale channels, Combust. Flame 155 (2008), 2-20.

[19] G. Pizza, C.E. Frouzakis, J. Mantzaras, A.G. Tomboulides, and K. Boulouchos, Threedimensional simulations of premixed hydrogen-air flames in microtubes, J. Fluid Mech. 658 (2010), 463-491.

[20] K. Maruta, J.K. Parc, K.C. Oh, T. Fujimori, S.S. Minaev, and R.V. Fursenko, Characteristics of microscale combustion in a narrow heated channel, Combust. Explos. Shock Waves 40 (2004), $516-523$.

[21] K. Maruta, T. Kataoka, N.I. Kim, S. Minaev, and R. Fursenko, Characteristics of combustion in a narrow channel with a temperature gradient, Proc. Combust. Inst. 30 (2005), 2429-2436.

[22] F. Richecoeur and D. Kyritsis, Experimental study of flame stabilization in low Reynolds and Dean number flows in curved mesoscale ducts, Proc. Combust. Inst. 30 (2005), 2419-2427.

[23] C.J. Evans and D.C. Kyritsis, Operational regimes of rich methane and propane/oxygen flames in mesoscale non-adiabatic ducts, Proc. Combust. Inst. 32 (2009), 3107-3114.

[24] P.D. Ronney, Analysis of non-adiabatic heat-recirculating combustors, Combust. Flame 135 (2003), 421-439.

[25] G. Pizza, J. Mantzaras, C.E. Frouzakis, A.G. Tomboulides, and K. Boulouchos, Suppression of combustion instabilities of premixed hydrogen/air flames in microchannels using heterogeneous reactions, Proc. Combust. Inst. 32 (2009), 3051-3058.

[26] P. Aghalayam, P.A. Bui, and D.G. Vlachos, The role of radical wall quenching in flame stability and wall heat flux: Hydrogen-air mixtures, Combust. Theory Model. 2 (1998), 515-530.

[27] B. Bai, Z. Chen, H. Zhang, and S. Chen, Flame propagation in a tube with wall quenching of radicals, Combust. Flame 160 (2013), 2810-2819.

[28] S.T. Lee and C.H. Tsai, Numerical investigation of steady laminar flame propagation in a circular tube, Combust. Flame 99 (1994), 484-490.

[29] N.I. Kim and K. Maruta, A numerical study on propagation of premixed flames in small tubes, Combust. Flame 146 (2006), 283-301.

[30] C.H. Tsai, The asymmetric behavior of steady laminar flame propagation in ducts, Combust. Sci. Technol. 180 (2008), 533-545.

[31] J.W. Dold, R.W. Thatcher, A. Omon-Arancibia, and J. Redman, From one-step to chainbranching premixed flame asymptotics, Proc. Combust. Inst. 29 (2002), 1519-1526.

[32] J.W. Dold, Premixed flames modelled with thermally sensitive intermediate branching kinetics, Combust. Theory Model. 11 (2007), 909-948.

[33] G.J. Sharpe, Thermal-diffusive instability of premixed flames for a simple chain-branching chemistry model with finite activation energy, SIAM J. Appl. Math. 70 (2009), 866-884.

[34] A.C. Fernández-Pello, Micropower generation using combustion: Issues and approaches, Proc. Combust. Inst. 29 (2002), 883-899.

[35] Y.B. Zel'dovich, Chain reactions in hot flames - an approximate theory for flame velocity, Kinetika i Kataliz 2 (1961), pp. 305-318 [English translation: Int. Chem. Engrg 2 (1962), pp. 227-235]. 
[36] A. Liñán, A theoretical analysis of premixed flame propagation with an isothermal chainbranching reaction, Tech. Rep. No. 1, INTA, Esteban Terradas, Madrid, 1971, USAFOSR Contract No. E00AR68-0031.

[37] F.A. Williams, Combustion Theory - The Fundamental Theory of Chemically Reacting Flow Systems, 2nd ed., Combustion Science and Engineering Series, F.A. Williams, ed., Benjamin/Cummings, Menlo Park, CA, 1985.

[38] A.L. Sánchez and F.A. Williams, Recent advances in understanding of flammability characteristics of hydrogen, Prog. Energy Combust. Sci. 41 (2014), 1-55.

[39] V.N. Kurdyumov and D. Fernández-Galisteo, Asymptotic structure of premixed flames for a simple chain-branching chemistry model with finite activation energy near the flammability limit, Combust. Flame 159 (2012), 3110-3118.

[40] D. Fernández-Galisteo, A.L. Sánchez, A. Liñán, and F.A. Williams, The hydrogen-air burning rate near the lean flammability limit, Combust. Theory Model. 13 (2009), 741-761.

[41] D. Fernández-Galisteo, A.L. Sánchez, A. Liñán, and F.A. Williams, One-step reduced kinetics for lean hydrogen-air deflagration, Combust. Flame 156 (2009), 985-996.

[42] V.V. Gubernov, A.V. Kolobov, A.A. Polezhaev, H.S. Sidhu, and G.N. Mercer, Pulsation instabilities of combustion waves in a chain-branching reaction model, Int. J. Bifur. Chaos 19 (2009), 873-887.

[43] D.W. Mikolaitis, Adiabatic flame speeds and the Zeldovich-Liñán model, Combust. Sci. Technol. 49 (1986), 277-288.

[44] R.J. Kee, J.F. Grcar, M.D. Smooke, and J.A. Miller, A Fortran program for modeling steady laminar one-dimensional premixed flames, Tech. Rep. SAND85-8240, Sandia National Laboratories, 1985.

[45] H. Pearlman, Excitability in high-Lewis number premixed gas combustion, Combust. Flame 109 (1997), 382-398.

[46] Chemical-kinetic mechanisms for combustion applications, San Diego Mechanism web page (2011). Available at http://combustion.ucsd.edu.

[47] N.M. Marinov, W.J. Pitz, C.K. Westbrook, A.M. Vincitore, M.J. Castaldi, S.M. Senkan, and C.F. Melius, Aromatic and polycyclic aromatic hydrocarbon formation in a laminar premixed n-butane flame, Combust. Flame 114 (1998), 192-213.

[48] Physical and Life Sciences Directorate, Lawrence Livermore National Laboratories. Available at https://www-pls.1lnl.gov/?url=science_and_technology-chemistry-combustion-ch4.

[49] D. Dunn-Rankin, Tulip flames: The shape of deflagrations in closed tubes, in Combustion Phenomena: Selected Mechanisms of Flame Formation, Propagation, and Extinction, J. Jarosinski and B. Veyssiere, eds., CRC Press, Taylor \& Francis Group, 2009, pp. 93-100.

[50] M. Sánchez-Sanz, D. Fernández-Galisteo, and V. Kurdyumov, Effects of equivalence ratio, Damköhler number, Lewis number and heat released on the stability of laminar premixed flames in microchannels, Combust. Flame. 161 (2014), 1282-1293. 